\title{
BOOK REVIEW: THERMAL CONVECTION: PATTERNS, EVOLUTION AND STABILITY
}

\author{
(Marcello Lappa, 2010, Wiley, Chichester, United Kingdom, 690 pp., \$240.00USD)
}

\section{J.A. Reizes}

University of Technology, P.O. Box 123, Broadway, Sydney, NSW 2007, Australia, E-mail: jreizes@eng.uts.edu.au

Despite the word "convection" appearing in the title, this excellent monograph is not a book on heat transfer. In fact, heat transfer is barely mentioned, with the only appearance of "heat transfer" in the index leading the reader to page 9 and the only graph involving the Nusselt number appearing on page 420 . This is not a criticism of the book since its focus is on the fluid mechanics of flows resulting from temperature differences. In fact, the subtitle "Patterns, Evolution and Stability" exactly describes the intent and content of the monograph. As such this text is still a necessary part of the armory of anyone seriously interested in thermally driven convection.

This book is not an introduction to convective flows caused by temperature differences, which is designed for a novice in the subject, but a rigorous voyage through the physics of many phenomena in thermal convection. As there are 49 pages of references, ${ }^{1}$ the information presented has been gleaned from a very thorough review of the theoretical, numerical, and experimental literature. The 13 chapters are organized in such a way that the reader is guided through the myriad of phenomena in an easily understood and mostly logical manner. Each chapter begins with an introduction to the particular phenomenon to be investigated, gradually introducing complexities (in particular, stability and possible multiple solutions, even chaos).

The first two chapters form an introduction that covers the derivation of the basic equations, basic concepts, methods of analysis, characteristic numbers, and scaling, while the following chapters are devoted to in-depth discussions of various phenomena under a generic descrip-

\footnotetext{
${ }^{1}$ It would have been helpful if the Reference list had given the page in the book where each reference is mentioned.
}

tor. Briefly, the remaining chapters are titled: "Examples of thermal fluid convection and pattern formation in nature and technology" (chapter 3); "The RayleighBénard problem" (chapter 4); "Thermal plumes and related flows" (chapter 5); "Systems heated from the side" (chapter 6); "Convection in inclined systems" (chapter 7); "Thermo-vibrational convection" (chapter 8); "Marangoni-Bénard convection" (chapter 9); "Thermocapillary convection" (chapter 10); "Mixed buoyancyMarangoni convection" (chapter 11); "Hybrid regimes with vibration" (chapter 12), and "Flow control by magnetic fields" (chapter 13).

Although very elegant, the derivation of the continuum conservation equations is considered from such a general point of view that readers who are not mathematically well equipped may find this part heavy going. However, as the author writes in the preface: "... the present book is devoted to a critical, focused and 'comparative' study of all these different types of thermal convection" and most of the remainder of the book is well presented and much easier to follow. The author's aim is clearly to develop a physical understanding of the flows being discussed by concentrating on presenting and discussing the evolution of the incredibly varying patterns that occur in convective flows and their stability.

Although there is a brief mention of numerical methods on pages $40-45$ of the first chapter, the problems associated with using computational approaches are not discussed. For example, on page 122 we find: "Historically, Jeffreys $(1926,1928)$ was the first (after some numerical difficulties) to yield the value of $\mathrm{Ra}_{\mathrm{cr}}=1707$ relevant to experiments using fluids [heated from below] between well-conducting solid parallel plates..." It is worth mentioning that Jeffreys reported that his solution 
was obtained-in 1926-by L. F. Richardson's method of finite differences, and his parenthetical comment "after some numerical difficulties" is the first indication of the problems associated with developing appropriate numerical methods and with finding suitable grids. Moreover, whichever computational technique might be used, there is no discussion of the care needed to ensure that reliable solutions have been obtained, particularly when bifurcations or instabilities are involved. Similar remarks apply to the difficulties of experimentation. The book is already over 600 pages long, and the inclusion of this material would have made it even longer. However, since such difficulties are rarely discussed, comments on these issues, however brief, would have been a useful contribution to the subject.

The range of scales presented is truly prodigiousfrom atmospheric and ocean phenomena, plate tectonics and convection in "other solar system bodies" to convection at low Rayleigh numbers in microgravity and on the microscale. Had the phenomena presented not been idealized by the use of the Boussinesq approximation in what might be termed "regular" geometries such as infinite horizontal and vertical layers, open and closed geometries, shallow and tall cavities (with "standard" boundary conditions such as adiabatic, isothermal, and conductive walls), the problem of a logical presentation would have been immeasurably more difficult and the length of the monograph greatly increased. This, of course, allows the author to free himself of the problems that occur from "real world" effects, which make quite difficult comparisons of numerically generated results with experimental data.

Although the restrictions on the temperature difference for which numerical results that use the assumption of the Boussinesq approximation are valid are briefly presented on page 65 (the limitation when dealing with liquids is actually much more severe than those mentioned on page 65 , which apply only to gases), the changes in flow behavior as the result of higher temperature differences are not discussed or even hinted at. Similarly, there is no mention of the difficulties of comparing numerical and experimental data, particularly the matching of boundary conditions used in numerical simulations to those actually employed in the matching experiment. This may require the solution of the conjugate problem involving the simulation of heat transfer to the environment-something that may be very difficult to do.

Even brief comments concerning such questions would have been useful. Otherwise, this is an excellent text, which I recommend for those seriously interested in thermally driven convection. 